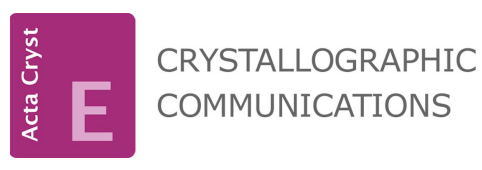

ISSN 2056-9890

Received 15 December 2015

Accepted 21 December 2015

Edited by H. Stoeckli-Evans, University of Neuchâtel, Switzerland

\section{Crystal structure of 7,8,9,10-tetrahydrobenzo[b]- naphtho[2,1-d]furan}

\author{
Zhongyuan $\mathrm{Wu}^{\mathrm{a}, \mathrm{b}}$ Manfred T. Reetz ${ }^{\mathrm{a}, \mathrm{b} *}$ and Klaus Harms ${ }^{\mathrm{a} *}$ \\ ${ }^{\text {a } F a c h b e r e i c h ~ C h e m i e, ~ P h i l i p p s-U n i v e r s i t a ̈ t, ~ H a n s-M e e r w e i n-S t r a s s e ~ 4, ~} 35032$ Marburg, Germany, and ${ }^{\mathbf{b}}$ Max-Planck- \\ Institut für Kohlenforschung, Kaiser-Wilhelm-Platz 1, 45470 Mülheim, Germany. *Correspondence e-mail: \\ reetz@mpi-muelheim.mpg.de, klaus.harms@chemie.uni-marburg.de
}

In the title compound, $\mathrm{C}_{16} \mathrm{H}_{14} \mathrm{O}$, the cyclohexene ring has a half-chair conformation. The mean plane, calculated through all non- $\mathrm{H}$ atoms of the molecule, except for the central $\mathrm{CH}_{2}$ atoms of the cyclohexene ring, which deviate by 0.340 (3) and -0.369 (3) $\AA$ from this mean plane, has an r.m.s. deviation of $0.012 \AA$. In the crystal, there are $\mathrm{C}-\mathrm{H} \cdots \pi$ contacts present, resulting in the formation of zigzag chains propagating along the [010] direction.

\section{Chemical context}

ords: crystal structure; Diels-Alder reaction; Friedel-Crafts reaction; furan; tetrahydrobenzonaphthofuran; $\mathrm{C}-\mathrm{H} \cdots \pi$ interactions

CCDC reference: 1429774

Supporting information: this article has supporting information at journals.iucr.org/e

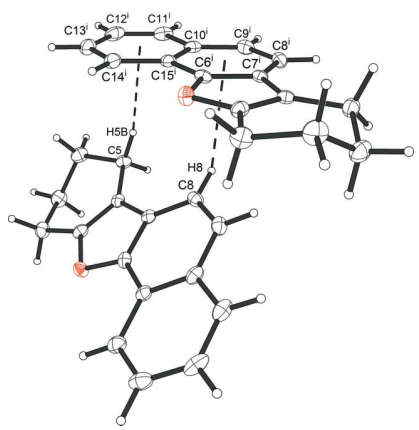

OPEN $\odot$ ACCESS
The interaction of Lewis acids with 1-naphthol $\mathbf{1}$ can be expected to induce metal coordination at the hydroxy function with concomitant increase in Brønsted-acidity (2) (Yamamoto \& Futatsugi, 2005; Goering, 1995). It is conceivable that the proton, once released from this intermediate $\mathbf{2}$, adds reversibly to the 4-position with formation of adduct $\mathbf{3}$, which is the Lewis acid coordinated form of the keto-tautomer of $\mathbf{1}$. Even if only minute amounts of $\mathbf{3}$ were to be formed, this intermediate should be a highly reactive dienophile in Diels-Alder reactions with such dienes as cyclohexadiene $\mathbf{4}$ leading to adduct 5 (see Scheme). Such a transformation implies dearomatization of 1-naphthol $\mathbf{1}$.

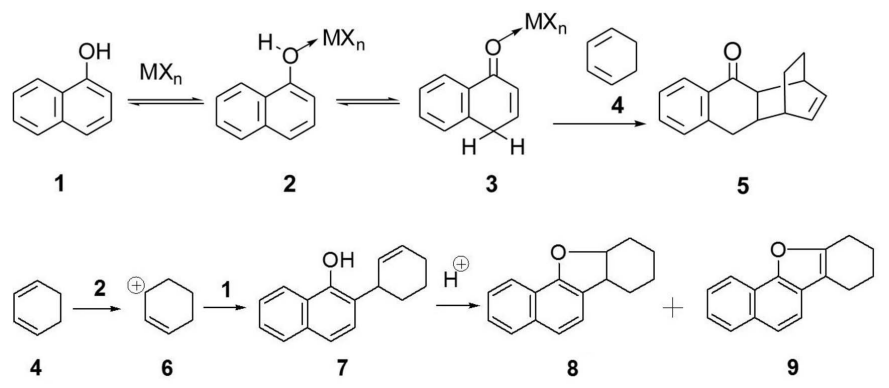

Alternatively, protonation of diene $\mathbf{4}$ leading to carbocation 6 would set the stage for Friedel-Crafts reaction with formation of the alkylation product $\mathbf{7}$, which could continue to react acid catalyzed, leading to adduct $\mathbf{8}$ and possibly to the aromatized furan product $\mathbf{9}$. In a previous study, Novák and coworkers reported the reaction of $\mathbf{1}$ with $\mathbf{4}$ in the presence of $\mathrm{TsOH} \cdot \mathrm{H}_{2} \mathrm{O}$ in boiling toluene $(26 \mathrm{~h})$ or at room temperature (7 d), furan derivative 9 being formed in 58\% yield, presumably via the intermediacy of $\mathbf{7}$ and $\mathbf{8}$ (Orovecz et al., 2003; Novák et al., 2000).

In exploratory experiments, we tested $\mathrm{Et}_{2} \mathrm{O} \cdot \mathrm{BF}_{3}, \mathrm{FeCl}_{3}$, $\mathrm{TiCl}_{4}$ and $\mathrm{ZrCl}_{4}$ as Lewis acids in the reaction of $\mathbf{1}$ and $\mathbf{4}$ at room temperature in $\mathrm{CH}_{2} \mathrm{Cl}_{2}$. Essentially only products derived from formal Friedel-Crafts alkylation were identified 


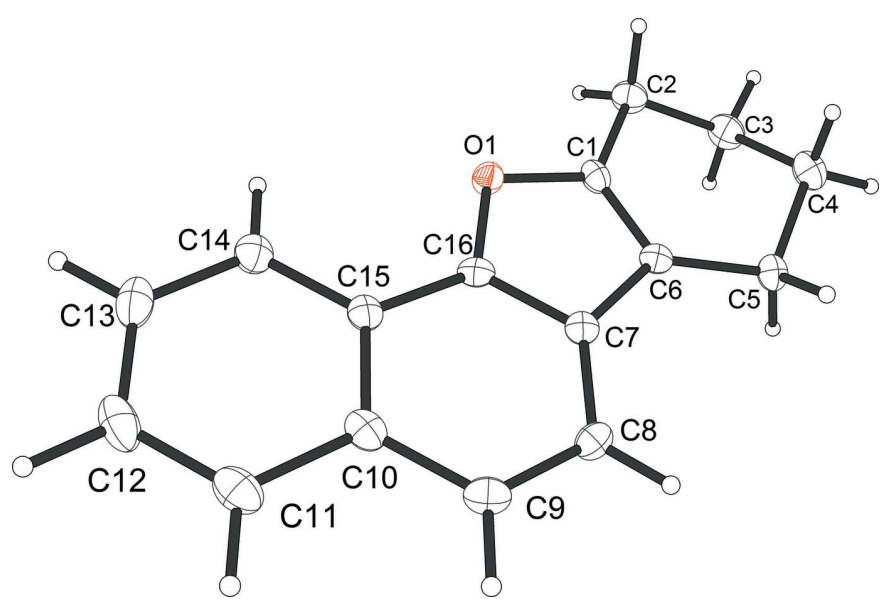

Figure 1

The molecular structure of compound $\mathbf{9}$, showing the atom labelling. Displacement ellipsoids are drawn at the $50 \%$ probability level.

following column chromatographic separation. Small amounts of unidentified compounds which could not be separated were also formed. A general protocol is provided. If a 2.5 -fold excess of cyclohexadiene $\mathbf{4}$ is used in these reactions, only small amounts of Friedel-Crafts products are formed (3-4\%). Rather, acid-mediated oligomerization of diene $\mathbf{4}$ occurs.

In contrast to the acidic conditions employed by Novák and coworkers, using the present protocol we isolated compound $\mathbf{8}$ and characterized it for the first time. We report herein on the crystal structure of the final product, furan 9.

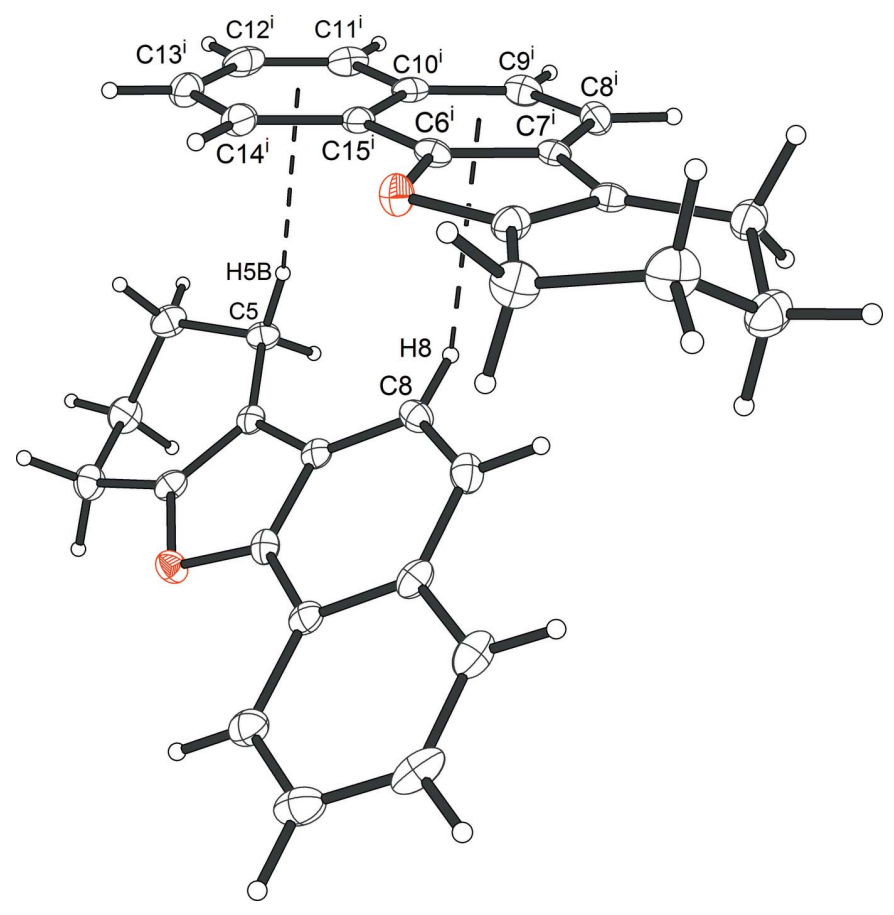

Figure 2

A view of the nearest $\mathrm{C}-\mathrm{H} \cdots$ ring centroid distances, shown as dashed lines [see Table 1; symmetry code: (i) $-x,-y+2, z-\frac{1}{2}$ ].
Table 1

Hydrogen-bond geometry $\left(\AA,^{\circ}\right)$.

$\mathrm{Cg} 3$ and $\mathrm{Cg} 4$ are the centroids of rings $\mathrm{C} 7-\mathrm{C} 10 / \mathrm{C} 15 / \mathrm{C} 16$ and $\mathrm{C} 10-\mathrm{C} 15$, respectively.

\begin{tabular}{lllll}
\hline$D-\mathrm{H} \cdots A$ & $D-\mathrm{H}$ & $\mathrm{H} \cdots A$ & $D \cdots A$ & $D-\mathrm{H} \cdots A$ \\
\hline $\mathrm{C} 5-\mathrm{H} 5 B \cdots C g 4^{\mathrm{i}}$ & 0.99 & 2.69 & $3.664(3)$ & 167 \\
$\mathrm{C} 8-\mathrm{H} 8 \cdots C g 3^{\mathrm{i}}$ & 0.95 & 2.93 & $3.650(3)$ & 134 \\
\hline
\end{tabular}

Symmetry code: (i) $-x,-y+2, z-\frac{1}{2}$.

\section{Structural commentary}

In the title compound 9, illustrated in Fig. 1, the cyclohexene ring (C1-C6) has a half-chair conformation. The mean plane, calculated through all non-hydrogen atoms of the molecule (O1/C1/C2/C5-C16), except atoms $\mathrm{C} 3$ and $\mathrm{C} 4$ of the cyclohexene ring that deviate by 0.340 (3) and -0.369 (3) $\AA$ from this mean plane, has an r.m.s. deviation of $0.012 \AA$. The other $\mathrm{C}$ and $\mathrm{O}$ atoms lie in this mean plane with a maximum deviation of -0.051 (3) $\AA$ for atom $\mathrm{C} 2$.

\section{Supramolecular features}

In the crystal of 9 , there are $\mathrm{C}-\mathrm{H} \cdots \pi$ contacts present (Table 1 and Fig. 2), but no classical hydrogen bonds and no $\pi-\pi$ interactions present. Intermolecular contacts thus appear to be limited to van der Waals interactions. The two rather short intermolecular $\mathrm{C}-\mathrm{H} \cdots$ ring centroid distances are: $\mathrm{H} 5 B \cdots$ centroid of ring $(\mathrm{C} 10-\mathrm{C} 15)=2.69 \AA$, H8 $\cdots$ centroid of ring $(\mathrm{C} 7-\mathrm{C} 10 / \mathrm{C} 15 / \mathrm{C} 16)=2.93 \AA$. These interactions result in the formation of zigzag chains propagating along the $b$-axis direction.

\section{Database survey}

Only one structure of a tetrahydrobenzonaphthofuran (Refcode PEBDAD; Scully \& Porco, 2012) is present in the current version 5.36 of the CSD (Groom \& Allen, 2014), and the cyclohexene ring also has a half-chair conformation.

\section{Synthesis and crystallization}

General Procedure: To a mixture of 1-naphthol (6.48 g, $45 \mathrm{mmol})$, catalyst $(2.25 \mathrm{mmol})$ in $\mathrm{CH}_{2} \mathrm{Cl}_{2}(10 \mathrm{ml}), 1,3$-cyclohexadiene $(0.7 \mathrm{ml}, 22.5 \mathrm{mmol})$ in $\mathrm{CH}_{2} \mathrm{Cl}_{2}(30 \mathrm{ml})$ was added drop wise, and the resulting solution was stirred at $273 \mathrm{~K}$ for $5 \mathrm{~h}$. After completion of the reaction (TLC) at room temperature, a cold aqueous solution of $\mathrm{NaHCO}_{3}(5 \%, 20 \mathrm{ml})$ was added and the mixture was extracted with $\mathrm{CH}_{2} \mathrm{Cl}_{2}(3 \times$ $10 \mathrm{ml})$. The organic extracts were washed with water $(2 \times 10 \mathrm{~mL})$ and dried over anhydrous $\mathrm{Na}_{2} \mathrm{SO}_{4}$, and concentrated in vacuum. The crude product was purified by silica column chromatography (petroleum ether) to give the desired product, which was identified by NMR spectroscopic comparison with authentic samples of $\mathbf{1}, \mathbf{2}$ and by X-ray diffraction analysis (Fig. 1). 
Table 2

Experimental details.

\begin{tabular}{|c|c|}
\hline \multicolumn{2}{|l|}{ Crystal data } \\
\hline Chemical formula & $\mathrm{C}_{16} \mathrm{H}_{14} \mathrm{O}$ \\
\hline$M_{\mathrm{r}}$ & 222.27 \\
\hline Crystal system, space group & Orthorhombic, Pna $2_{1}$ \\
\hline Temperature (K) & 100 \\
\hline$a, b, c(\AA)$ & $13.8369(9), 12.2202(8), 6.8468(4)$ \\
\hline$V\left(\AA^{3}\right)$ & $1157.72(13)$ \\
\hline$Z$ & 4 \\
\hline Radiation type & Мо $K \alpha$ \\
\hline$\mu\left(\mathrm{mm}^{-1}\right)$ & 0.08 \\
\hline Crystal size (mm) & $0.16 \times 0.05 \times 0.04$ \\
\hline \multicolumn{2}{|l|}{ Data collection } \\
\hline Diffractometer & Bruker D8 QUEST area detector \\
\hline Absorption correction & $\begin{array}{l}\text { Multi-scan (SADABS; Bruker, } \\
\text { 2014) }\end{array}$ \\
\hline$T_{\min }, T_{\max }$ & $0.94,1.00$ \\
\hline $\begin{array}{l}\text { No. of measured, independent and } \\
\text { observed }[I>2 \sigma(I)] \text { reflections }\end{array}$ & $5983,2024,1808$ \\
\hline$R_{\text {int }}$ & 0.038 \\
\hline$(\sin \theta / \lambda)_{\max }\left(\AA^{-1}\right)$ & 0.601 \\
\hline \multicolumn{2}{|l|}{ Refinement } \\
\hline$R\left[F^{2}>2 \sigma\left(F^{2}\right)\right], w R\left(F^{2}\right), S$ & $0.038,0.081,1.09$ \\
\hline No. of reflections & 2024 \\
\hline No. of parameters & 154 \\
\hline No. of restraints & 1 \\
\hline H-atom treatment & $\mathrm{H}$-atom parameters constrained \\
\hline$\Delta \rho_{\max }, \Delta \rho_{\min }\left(\mathrm{e} \AA^{-3}\right)$ & $0.16,-0.24$ \\
\hline
\end{tabular}

Computer programs: APEX2 and SAINT (Bruker, 2014), SHELXT (Sheldrick, 2015a), SHELXL (Sheldrick, 2015b), DIAMOND (Brandenburg, 2006), publCIF (Westrip, 2010) and PLATON (Spek, 2009).

Compound 8: ${ }^{1} \mathrm{H}$ NMR (300 $\mathrm{MHz}, \mathrm{CDCl}_{3}$, p.p.m.): $\delta 1.19$ $1.27(m, 1 \mathrm{H}), 1.34-1.48(m, 4 \mathrm{H}), 1.69-1.84(m, 2 \mathrm{H}), 1.92-2.02$ $(m, 1 \mathrm{H}), 3.17-3.24(m, 1 \mathrm{H}), 4.71--4.77(m, 1 \mathrm{H}), 7.16-7.18(m$, $1 \mathrm{H}), 7.24-7.32(m, 3 \mathrm{H}), 7.66-7.69(m, 1 \mathrm{H}), 7.87-7.90(m, 1 \mathrm{H})$;

${ }^{13} \mathrm{C}$ NMR (300 MHz, $\mathrm{CDCl}_{3}$, p.p.m.): $\delta$ 20.50, 21.86, 27.64,
$28.38,41.41,83.44,120.16,121.16,121.60,121.92,125.13$, 125.48, 126.55, 128.01, 134.11, 155.07 .

High Resolution Mass Spectrum: $\left(M+\mathrm{H}^{+}\right)$calculated for $\mathrm{C}_{16} \mathrm{H}_{16} \mathrm{O} 225.1274$; found $\left(M+\mathrm{H}^{+}\right) 225.1275$.

\section{Refinement}

Crystal data, data collection and structure refinement details are summarized in Table $2 . \mathrm{H}$ atoms were located in difference Fourier maps, but subsequently included in the refinement using a riding model: $\mathrm{C}-\mathrm{H}=0.95-0.99 \AA$ with $U_{\text {iso }}(\mathrm{H})=$ $1.2 U_{\text {eq }}(\mathrm{C})$.

\section{Acknowledgements}

This work was supported by the Max-Plank Society, Germany.

\section{References}

Brandenburg, K. (2006). DIAMOND. Crystal Impact GbR, Bonn, Germany.

Bruker (2014). APEX2, SAINT and SADABS. Bruker AXS Inc., Madison, Wisconsin, USA.

Goering, B. K. (1995). PhD dissertation, Cornell University, Ithaca, USA.

Groom, C. R. \& Allen, F. H. (2014). Angew. Chem. Int. Ed. 53, 662671.

Novák, L., Kovács, P., Kolonits, P., Orovecz, O., Fekete, J. \& Szántay, C. (2000). Synthesis, 6, 809-812.

Orovecz, O., Kovács, P., Kolonits, P., Kaleta, Z., Párkányi, L., Szabó, É. \& Novák, L. (2003). Synthesis, 7, 1043-1048.

Scully, S. S. \& Porco, J. A. Jr (2012). Org. Lett. 14, 2646-2649.

Sheldrick, G. M. (2015a). Acta Cryst. A71, 3-8.

Sheldrick, G. M. (2015b). Acta Cryst. C71, 3-8.

Spek, A. L. (2009). Acta Cryst. D65, 148-155.

Westrip, S. P. (2010). J. Appl. Cryst. 43, 920-925.

Yamamoto, H. \& Futatsugi, K. (2005). Angew. Chem. Int. Ed. 44, 1924-1942. 


\section{supporting information}

Acta Cryst. (2016). E72, 106-108 [doi:10.1107/S2056989015024512]

\section{Crystal structure of 7,8,9,10-tetrahydrobenzo[b]naphtho[2,1-d] furan}

\section{Zhongyuan Wu, Manfred T. Reetz and Klaus Harms}

Computing details

Data collection: APEX2 (Bruker, 2014); cell refinement: SAINT (Bruker, 2014); data reduction: SAINT (Bruker, 2014); program(s) used to solve structure: SHELXT (Sheldrick, 2015a); program(s) used to refine structure: SHELXL (Sheldrick, 2015b); molecular graphics: DIAMOND (Brandenburg, 2006); software used to prepare material for publication: publCIF (Westrip, 2010) and PLATON (Spek, 2009).

7,8,9,10-Tetrahydrobenzo $[b]$ naphtho $[2,1-d]$ furan

Crystal data

$\mathrm{C}_{16} \mathrm{H}_{14} \mathrm{O}$

$M_{r}=222.27$

Orthorhombic, Pna2 1

$a=13.8369$ (9) $\AA$

$b=12.2202(8) \AA$

$c=6.8468(4) \AA$

$V=1157.72(13) \AA^{3}$

$Z=4$

$F(000)=472$

\section{Data collection}

Bruker D8 QUEST area-detector diffractometer

Radiation source: microfocus sealed X-ray tube Detector resolution: 7.9 pixels $\mathrm{mm}^{-1}$

$\omega$ and $\varphi$ scans

Absorption correction: multi-scan

(SADABS; Bruker, 2014)

$T_{\min }=0.94, T_{\max }=1.00$

Refinement

Refinement on $F^{2}$

Least-squares matrix: full

$R\left[F^{2}>2 \sigma\left(F^{2}\right)\right]=0.038$

$w R\left(F^{2}\right)=0.081$

$S=1.09$

2024 reflections

154 parameters

1 restraint

Primary atom site location: structure-invariant direct methods
$D_{\mathrm{x}}=1.275 \mathrm{Mg} \mathrm{m}^{-3}$

Mo $K \alpha$ radiation, $\lambda=0.71073 \AA$

Cell parameters from 2867 reflections

$\theta=2.2-25.2^{\circ}$

$\mu=0.08 \mathrm{~mm}^{-1}$

$T=100 \mathrm{~K}$

Prism, colourless

$0.16 \times 0.05 \times 0.04 \mathrm{~mm}$

5983 measured reflections

2024 independent reflections

1808 reflections with $I>2 \sigma(I)$

$R_{\text {int }}=0.038$

$\theta_{\max }=25.3^{\circ}, \theta_{\min }=2.2^{\circ}$

$h=-16 \rightarrow 16$

$k=-14 \rightarrow 14$

$l=-8 \rightarrow 8$

Secondary atom site location: difference Fourier map

Hydrogen site location: inferred from neighbouring sites

$\mathrm{H}$-atom parameters constrained

$w=1 /\left[\sigma^{2}\left(F_{\mathrm{o}}^{2}\right)+(0.0435 P)^{2}+0.0066 P\right]$

where $P=\left(F_{\mathrm{o}}^{2}+2 F_{\mathrm{c}}{ }^{2}\right) / 3$

$(\Delta / \sigma)_{\max }<0.001$

$\Delta \rho_{\max }=0.16 \mathrm{e} \AA^{-3}$

$\Delta \rho_{\min }=-0.24$ e $\AA^{-3}$ 


\section{Special details}

Geometry. All esds (except the esd in the dihedral angle between two 1.s. planes) are estimated using the full covariance matrix. The cell esds are taken into account individually in the estimation of esds in distances, angles and torsion angles; correlations between esds in cell parameters are only used when they are defined by crystal symmetry. An approximate (isotropic) treatment of cell esds is used for estimating esds involving l.s. planes.

Fractional atomic coordinates and isotropic or equivalent isotropic displacement parameters $\left(\AA^{2}\right)$

\begin{tabular}{|c|c|c|c|c|}
\hline & $x$ & $y$ & $z$ & $U_{\text {iso }} * / U_{\text {eq }}$ \\
\hline $\mathrm{C} 1$ & $0.03747(16)$ & $0.7566(2)$ & $0.3738(4)$ & $0.0159(5)$ \\
\hline $\mathrm{O} 1$ & $0.09729(11)$ & $0.83253(13)$ & $0.4640(2)$ & $0.0165(4)$ \\
\hline $\mathrm{C} 2$ & 0.00207 (19) & $0.6599(2)$ & $0.4844(4)$ & $0.0207(6)$ \\
\hline $\mathrm{H} 2 \mathrm{~A}$ & -0.0492 & 0.6822 & 0.5770 & $0.025^{*}$ \\
\hline $\mathrm{H} 2 \mathrm{~B}$ & 0.0557 & 0.6267 & 0.5596 & $0.025^{*}$ \\
\hline $\mathrm{C} 3$ & $-0.03802(19)$ & $0.5773(2)$ & $0.3369(4)$ & $0.0237(6)$ \\
\hline $\mathrm{H} 3 \mathrm{~A}$ & -0.0767 & 0.5215 & 0.4070 & $0.028^{*}$ \\
\hline $\mathrm{H} 3 \mathrm{~B}$ & 0.0164 & 0.5395 & 0.2716 & $0.028^{*}$ \\
\hline $\mathrm{C} 4$ & $-0.10145(19)$ & $0.6329(2)$ & $0.1823(4)$ & $0.0232(6)$ \\
\hline $\mathrm{H} 4 \mathrm{~A}$ & -0.1300 & 0.5763 & 0.0964 & $0.028^{*}$ \\
\hline H4B & -0.1551 & 0.6719 & 0.2480 & $0.028^{*}$ \\
\hline $\mathrm{C} 5$ & $-0.04412(18)$ & $0.7140(2)$ & $0.0581(4)$ & $0.0179(6)$ \\
\hline $\mathrm{H} 5 \mathrm{~A}$ & -0.0036 & 0.6738 & -0.0369 & $0.021^{*}$ \\
\hline H5B & -0.0891 & 0.7616 & -0.0156 & $0.021^{*}$ \\
\hline C6 & $0.01857(16)$ & $0.78260(19)$ & $0.1870(4)$ & $0.0138(5)$ \\
\hline $\mathrm{C} 7$ & $0.06900(16)$ & $0.8841(2)$ & $0.1498(3)$ & $0.0137(5)$ \\
\hline $\mathrm{C} 8$ & $0.07806(17)$ & $0.9549(2)$ & $-0.0120(4)$ & $0.0165(6)$ \\
\hline $\mathrm{H} 8$ & 0.0475 & 0.9381 & -0.1326 & $0.020^{*}$ \\
\hline C9 & $0.13190(18)$ & $1.0484(2)$ & $0.0084(4)$ & $0.0187(6)$ \\
\hline H9 & 0.1371 & 1.0973 & -0.0991 & $0.022 *$ \\
\hline $\mathrm{C} 10$ & $0.18051(17)$ & 1.07448 (19) & $0.1866(4)$ & $0.0171(6)$ \\
\hline C11 & $0.23745(18)$ & $1.1700(2)$ & $0.2054(4)$ & $0.0215(6)$ \\
\hline H11 & 0.2441 & 1.2179 & 0.0969 & $0.026^{*}$ \\
\hline $\mathrm{C} 12$ & $0.28309(18)$ & 1.1947 (2) & $0.3768(4)$ & $0.0243(7)$ \\
\hline H12 & 0.3209 & 1.2593 & 0.3862 & $0.029^{*}$ \\
\hline $\mathrm{C} 13$ & $0.27444(18)$ & $1.1254(2)$ & $0.5383(4)$ & $0.0234(6)$ \\
\hline H13 & 0.3060 & 1.1438 & 0.6571 & $0.028^{*}$ \\
\hline C14 & $0.22067(17)$ & $1.0308(2)$ & $0.5271(4)$ & $0.0191(6)$ \\
\hline H14 & 0.2157 & 0.9837 & 0.6371 & $0.023^{*}$ \\
\hline $\mathrm{C} 15$ & $0.17295(17)$ & $1.0040(2)$ & $0.3507(4)$ & $0.0151(5)$ \\
\hline $\mathrm{C} 16$ & $0.11541(17)$ & $0.9100(2)$ & $0.3225(3)$ & $0.0141(5)$ \\
\hline
\end{tabular}

Atomic displacement parameters $\left(\AA^{2}\right)$

\begin{tabular}{lllllll}
\hline & $U^{11}$ & $U^{22}$ & $U^{33}$ & $U^{12}$ & $U^{13}$ & $U^{23}$ \\
\hline C1 & $0.0135(11)$ & $0.0131(13)$ & $0.0211(13)$ & $-0.0013(10)$ & $0.0009(11)$ & $-0.0021(11)$ \\
O1 & $0.0202(8)$ & $0.0147(9)$ & $0.0144(8)$ & $-0.0019(8)$ & $-0.0021(7)$ & $0.0019(7)$ \\
C2 & $0.0232(13)$ & $0.0180(14)$ & $0.0210(14)$ & $-0.0019(11)$ & $0.0019(12)$ & $0.0042(12)$ \\
C3 & $0.0263(14)$ & $0.0171(14)$ & $0.0277(14)$ & $-0.0048(12)$ & $0.0018(13)$ & $0.0035(12)$
\end{tabular}




\begin{tabular}{lllllll} 
C4 & $0.0197(13)$ & $0.0213(15)$ & $0.0286(15)$ & $-0.0056(12)$ & $-0.0006(12)$ & $-0.0007(13)$ \\
C5 & $0.0164(12)$ & $0.0169(14)$ & $0.0203(13)$ & $-0.0010(11)$ & $-0.0023(11)$ & $-0.0023(12)$ \\
C6 & $0.0126(11)$ & $0.0128(13)$ & $0.0159(12)$ & $0.0037(10)$ & $0.0024(10)$ & $-0.0007(11)$ \\
C7 & $0.0119(12)$ & $0.0132(13)$ & $0.0158(12)$ & $0.0041(10)$ & $0.0022(11)$ & $-0.0027(11)$ \\
C8 & $0.0185(12)$ & $0.0172(14)$ & $0.0140(12)$ & $0.0036(11)$ & $-0.0010(11)$ & $-0.0012(11)$ \\
C9 & $0.0200(12)$ & $0.0174(13)$ & $0.0186(13)$ & $0.0036(11)$ & $0.0040(11)$ & $0.0030(12)$ \\
C10 & $0.0127(11)$ & $0.0153(13)$ & $0.0233(14)$ & $0.0024(10)$ & $0.0046(11)$ & $-0.0019(12)$ \\
C11 & $0.0168(13)$ & $0.0174(15)$ & $0.0303(15)$ & $0.0005(11)$ & $0.0056(13)$ & $0.0015(13)$ \\
C12 & $0.0140(12)$ & $0.0199(15)$ & $0.0391(17)$ & $-0.0041(11)$ & $0.0037(13)$ & $-0.0069(14)$ \\
C13 & $0.0167(12)$ & $0.0257(16)$ & $0.0278(15)$ & $-0.0006(12)$ & $-0.0023(12)$ & $-0.0106(14)$ \\
C14 & $0.0149(12)$ & $0.0209(14)$ & $0.0216(13)$ & $0.0012(11)$ & $0.0002(11)$ & $-0.0020(13)$ \\
C15 & $0.0115(11)$ & $0.0159(13)$ & $0.0180(12)$ & $0.0020(10)$ & $0.0021(10)$ & $-0.0026(11)$ \\
C16 & $0.0155(12)$ & $0.0117(13)$ & $0.0152(13)$ & $0.0035(10)$ & $0.0039(11)$ & $0.0003(11)$ \\
\hline
\end{tabular}

Geometric parameters $\left(\AA,{ }^{o}\right)$

\begin{tabular}{|c|c|c|c|}
\hline $\mathrm{C} 1-\mathrm{C} 6$ & $1.343(3)$ & $\mathrm{C} 7-\mathrm{C} 16$ & $1.383(3)$ \\
\hline $\mathrm{C} 1-\mathrm{O} 1$ & $1.388(3)$ & $\mathrm{C} 7-\mathrm{C} 8$ & $1.411(3)$ \\
\hline $\mathrm{C} 1-\mathrm{C} 2$ & $1.486(3)$ & $\mathrm{C} 8-\mathrm{C} 9$ & $1.371(3)$ \\
\hline $\mathrm{O} 1-\mathrm{C} 16$ & $1.377(3)$ & $\mathrm{C} 8-\mathrm{H} 8$ & 0.9500 \\
\hline $\mathrm{C} 2-\mathrm{C} 3$ & $1.532(4)$ & $\mathrm{C} 9-\mathrm{C} 10$ & $1.429(4)$ \\
\hline $\mathrm{C} 2-\mathrm{H} 2 \mathrm{~A}$ & 0.9900 & $\mathrm{C} 9-\mathrm{H} 9$ & 0.9500 \\
\hline $\mathrm{C} 2-\mathrm{H} 2 \mathrm{~B}$ & 0.9900 & $\mathrm{C} 10-\mathrm{C} 11$ & $1.414(4)$ \\
\hline $\mathrm{C} 3-\mathrm{C} 4$ & $1.534(4)$ & $\mathrm{C} 10-\mathrm{C} 15$ & $1.419(3)$ \\
\hline $\mathrm{C} 3-\mathrm{H} 3 \mathrm{~A}$ & 0.9900 & $\mathrm{C} 11-\mathrm{C} 12$ & $1.366(4)$ \\
\hline С $3-\mathrm{H} 3 \mathrm{~B}$ & 0.9900 & $\mathrm{C} 11-\mathrm{H} 11$ & 0.9500 \\
\hline $\mathrm{C} 4-\mathrm{C} 5$ & $1.528(4)$ & $\mathrm{C} 12-\mathrm{C} 13$ & $1.398(4)$ \\
\hline $\mathrm{C} 4-\mathrm{H} 4 \mathrm{~A}$ & 0.9900 & $\mathrm{C} 12-\mathrm{H} 12$ & 0.9500 \\
\hline $\mathrm{C} 4-\mathrm{H} 4 \mathrm{~B}$ & 0.9900 & $\mathrm{C} 13-\mathrm{C} 14$ & $1.377(3)$ \\
\hline $\mathrm{C} 5-\mathrm{C} 6$ & $1.494(3)$ & $\mathrm{C} 13-\mathrm{H} 13$ & 0.9500 \\
\hline $\mathrm{C} 5-\mathrm{H} 5 \mathrm{~A}$ & 0.9900 & $\mathrm{C} 14-\mathrm{C} 15$ & $1.415(4)$ \\
\hline C5-H5B & 0.9900 & $\mathrm{C} 14-\mathrm{H} 14$ & 0.9500 \\
\hline $\mathrm{C} 6-\mathrm{C} 7$ & $1.446(3)$ & $\mathrm{C} 15-\mathrm{C} 16$ & $1.411(3)$ \\
\hline $\mathrm{C} 6-\mathrm{C} 1-\mathrm{O} 1$ & $112.4(2)$ & $\mathrm{C} 16-\mathrm{C} 7-\mathrm{C} 8$ & $119.4(2)$ \\
\hline $\mathrm{C} 6-\mathrm{C} 1-\mathrm{C} 2$ & $127.5(2)$ & $\mathrm{C} 16-\mathrm{C} 7-\mathrm{C} 6$ & $105.6(2)$ \\
\hline $\mathrm{O} 1-\mathrm{C} 1-\mathrm{C} 2$ & $120.1(2)$ & $\mathrm{C} 8-\mathrm{C} 7-\mathrm{C} 6$ & $135.0(2)$ \\
\hline $\mathrm{C} 16-\mathrm{O} 1-\mathrm{C} 1$ & $104.80(18)$ & $\mathrm{C} 9-\mathrm{C} 8-\mathrm{C} 7$ & $118.6(2)$ \\
\hline $\mathrm{C} 1-\mathrm{C} 2-\mathrm{C} 3$ & $107.9(2)$ & $\mathrm{C} 9-\mathrm{C} 8-\mathrm{H} 8$ & 120.7 \\
\hline $\mathrm{C} 1-\mathrm{C} 2-\mathrm{H} 2 \mathrm{~A}$ & 110.1 & $\mathrm{C} 7-\mathrm{C} 8-\mathrm{H} 8$ & 120.7 \\
\hline $\mathrm{C} 3-\mathrm{C} 2-\mathrm{H} 2 \mathrm{~A}$ & 110.1 & $\mathrm{C} 8-\mathrm{C} 9-\mathrm{C} 10$ & $121.9(2)$ \\
\hline $\mathrm{C} 1-\mathrm{C} 2-\mathrm{H} 2 \mathrm{~B}$ & 110.1 & $\mathrm{C} 8-\mathrm{C} 9-\mathrm{H} 9$ & 119.1 \\
\hline $\mathrm{C} 3-\mathrm{C} 2-\mathrm{H} 2 \mathrm{~B}$ & 110.1 & $\mathrm{C} 10-\mathrm{C} 9-\mathrm{H} 9$ & 119.1 \\
\hline $\mathrm{H} 2 \mathrm{~A}-\mathrm{C} 2-\mathrm{H} 2 \mathrm{~B}$ & 108.4 & $\mathrm{C} 11-\mathrm{C} 10-\mathrm{C} 15$ & $118.0(2)$ \\
\hline $\mathrm{C} 2-\mathrm{C} 3-\mathrm{C} 4$ & $111.7(2)$ & $\mathrm{C} 11-\mathrm{C} 10-\mathrm{C} 9$ & $121.6(2)$ \\
\hline $\mathrm{C} 2-\mathrm{C} 3-\mathrm{H} 3 \mathrm{~A}$ & 109.3 & $\mathrm{C} 15-\mathrm{C} 10-\mathrm{C} 9$ & $120.4(2)$ \\
\hline $\mathrm{C} 4-\mathrm{C} 3-\mathrm{H} 3 \mathrm{~A}$ & 109.3 & $\mathrm{C} 12-\mathrm{C} 11-\mathrm{C} 10$ & $121.2(3)$ \\
\hline $\mathrm{C} 2-\mathrm{C} 3-\mathrm{H} 3 \mathrm{~B}$ & 109.3 & $\mathrm{C} 12-\mathrm{C} 11-\mathrm{H} 11$ & 119.4 \\
\hline
\end{tabular}




\begin{tabular}{|c|c|c|c|}
\hline $\mathrm{C} 4-\mathrm{C} 3-\mathrm{H} 3 \mathrm{~B}$ & 109.3 & $\mathrm{C} 10-\mathrm{C} 11-\mathrm{H} 11$ & 119.4 \\
\hline $\mathrm{H} 3 \mathrm{~A}-\mathrm{C} 3-\mathrm{H} 3 \mathrm{~B}$ & 107.9 & $\mathrm{C} 11-\mathrm{C} 12-\mathrm{C} 13$ & $120.4(2)$ \\
\hline $\mathrm{C} 5-\mathrm{C} 4-\mathrm{C} 3$ & $112.0(2)$ & $\mathrm{C} 11-\mathrm{C} 12-\mathrm{H} 12$ & 119.8 \\
\hline $\mathrm{C} 5-\mathrm{C} 4-\mathrm{H} 4 \mathrm{~A}$ & 109.2 & $\mathrm{C} 13-\mathrm{C} 12-\mathrm{H} 12$ & 119.8 \\
\hline $\mathrm{C} 3-\mathrm{C} 4-\mathrm{H} 4 \mathrm{~A}$ & 109.2 & $\mathrm{C} 14-\mathrm{C} 13-\mathrm{C} 12$ & $120.7(3)$ \\
\hline $\mathrm{C} 5-\mathrm{C} 4-\mathrm{H} 4 \mathrm{~B}$ & 109.2 & $\mathrm{C} 14-\mathrm{C} 13-\mathrm{H} 13$ & 119.6 \\
\hline $\mathrm{C} 3-\mathrm{C} 4-\mathrm{H} 4 \mathrm{~B}$ & 109.2 & $\mathrm{C} 12-\mathrm{C} 13-\mathrm{H} 13$ & 119.6 \\
\hline $\mathrm{H} 4 \mathrm{~A}-\mathrm{C} 4-\mathrm{H} 4 \mathrm{~B}$ & 107.9 & $\mathrm{C} 13-\mathrm{C} 14-\mathrm{C} 15$ & $119.6(2)$ \\
\hline $\mathrm{C} 6-\mathrm{C} 5-\mathrm{C} 4$ & $109.7(2)$ & $\mathrm{C} 13-\mathrm{C} 14-\mathrm{H} 14$ & 120.2 \\
\hline $\mathrm{C} 6-\mathrm{C} 5-\mathrm{H} 5 \mathrm{~A}$ & 109.7 & $\mathrm{C} 15-\mathrm{C} 14-\mathrm{H} 14$ & 120.2 \\
\hline $\mathrm{C} 4-\mathrm{C} 5-\mathrm{H} 5 \mathrm{~A}$ & 109.7 & $\mathrm{C} 16-\mathrm{C} 15-\mathrm{C} 14$ & $124.6(2)$ \\
\hline $\mathrm{C} 6-\mathrm{C} 5-\mathrm{H} 5 \mathrm{~B}$ & 109.7 & $\mathrm{C} 16-\mathrm{C} 15-\mathrm{C} 10$ & $115.3(2)$ \\
\hline $\mathrm{C} 4-\mathrm{C} 5-\mathrm{H} 5 \mathrm{~B}$ & 109.7 & $\mathrm{C} 14-\mathrm{C} 15-\mathrm{C} 10$ & $120.1(2)$ \\
\hline $\mathrm{H} 5 \mathrm{~A}-\mathrm{C} 5-\mathrm{H} 5 \mathrm{~B}$ & 108.2 & $\mathrm{O} 1-\mathrm{C} 16-\mathrm{C} 7$ & $111.1(2)$ \\
\hline $\mathrm{C} 1-\mathrm{C} 6-\mathrm{C} 7$ & $106.1(2)$ & $\mathrm{O} 1-\mathrm{C} 16-\mathrm{C} 15$ & $124.5(2)$ \\
\hline $\mathrm{C} 1-\mathrm{C} 6-\mathrm{C} 5$ & $122.8(2)$ & $\mathrm{C} 7-\mathrm{C} 16-\mathrm{C} 15$ & $124.4(2)$ \\
\hline $\mathrm{C} 7-\mathrm{C} 6-\mathrm{C} 5$ & $131.1(2)$ & & \\
\hline $\mathrm{C} 6-\mathrm{C} 1-\mathrm{O} 1-\mathrm{C} 16$ & $0.3(2)$ & $\mathrm{C} 15-\mathrm{C} 10-\mathrm{C} 11-\mathrm{C} 12$ & $-0.7(4)$ \\
\hline $\mathrm{C} 2-\mathrm{C} 1-\mathrm{O} 1-\mathrm{C} 16$ & $-179.7(2)$ & $\mathrm{C} 9-\mathrm{C} 10-\mathrm{C} 11-\mathrm{C} 12$ & $179.5(2)$ \\
\hline $\mathrm{C} 6-\mathrm{C} 1-\mathrm{C} 2-\mathrm{C} 3$ & $15.0(3)$ & $\mathrm{C} 10-\mathrm{C} 11-\mathrm{C} 12-\mathrm{C} 13$ & $0.1(4)$ \\
\hline $\mathrm{O} 1-\mathrm{C} 1-\mathrm{C} 2-\mathrm{C} 3$ & $-165.0(2)$ & $\mathrm{C} 11-\mathrm{C} 12-\mathrm{C} 13-\mathrm{C} 14$ & $0.6(4)$ \\
\hline $\mathrm{C} 1-\mathrm{C} 2-\mathrm{C} 3-\mathrm{C} 4$ & $-44.6(3)$ & $\mathrm{C} 12-\mathrm{C} 13-\mathrm{C} 14-\mathrm{C} 15$ & $-0.6(4)$ \\
\hline $\mathrm{C} 2-\mathrm{C} 3-\mathrm{C} 4-\mathrm{C} 5$ & $63.3(3)$ & $\mathrm{C} 13-\mathrm{C} 14-\mathrm{C} 15-\mathrm{C} 16$ & $-179.5(2)$ \\
\hline $\mathrm{C} 3-\mathrm{C} 4-\mathrm{C} 5-\mathrm{C} 6$ & $-45.0(3)$ & $\mathrm{C} 13-\mathrm{C} 14-\mathrm{C} 15-\mathrm{C} 10$ & $-0.1(4)$ \\
\hline $\mathrm{O} 1-\mathrm{C} 1-\mathrm{C} 6-\mathrm{C} 7$ & $-0.5(3)$ & $\mathrm{C} 11-\mathrm{C} 10-\mathrm{C} 15-\mathrm{C} 16$ & $-179.8(2)$ \\
\hline $\mathrm{C} 2-\mathrm{C} 1-\mathrm{C} 6-\mathrm{C} 7$ & $179.5(2)$ & $\mathrm{C} 9-\mathrm{C} 10-\mathrm{C} 15-\mathrm{C} 16$ & $0.0(3)$ \\
\hline $\mathrm{O} 1-\mathrm{C} 1-\mathrm{C} 6-\mathrm{C} 5$ & $-179.9(2)$ & $\mathrm{C} 11-\mathrm{C} 10-\mathrm{C} 15-\mathrm{C} 14$ & $0.7(3)$ \\
\hline $\mathrm{C} 2-\mathrm{C} 1-\mathrm{C} 6-\mathrm{C} 5$ & $0.0(4)$ & $\mathrm{C} 9-\mathrm{C} 10-\mathrm{C} 15-\mathrm{C} 14$ & $-179.5(2)$ \\
\hline $\mathrm{C} 4-\mathrm{C} 5-\mathrm{C} 6-\mathrm{C} 1$ & $14.8(3)$ & $\mathrm{C} 1-\mathrm{O} 1-\mathrm{C} 16-\mathrm{C} 7$ & $0.0(2)$ \\
\hline $\mathrm{C} 4-\mathrm{C} 5-\mathrm{C} 6-\mathrm{C} 7$ & $-164.5(2)$ & $\mathrm{C} 1-\mathrm{O} 1-\mathrm{C} 16-\mathrm{C} 15$ & $179.4(2)$ \\
\hline $\mathrm{C} 1-\mathrm{C} 6-\mathrm{C} 7-\mathrm{C} 16$ & $0.4(2)$ & $\mathrm{C} 8-\mathrm{C} 7-\mathrm{C} 16-\mathrm{O} 1$ & $179.21(19)$ \\
\hline $\mathrm{C} 5-\mathrm{C} 6-\mathrm{C} 7-\mathrm{C} 16$ & $179.8(2)$ & $\mathrm{C} 6-\mathrm{C} 7-\mathrm{C} 16-\mathrm{O} 1$ & $-0.2(3)$ \\
\hline $\mathrm{C} 1-\mathrm{C} 6-\mathrm{C} 7-\mathrm{C} 8$ & $-178.9(3)$ & $\mathrm{C} 8-\mathrm{C} 7-\mathrm{C} 16-\mathrm{C} 15$ & $-0.2(3)$ \\
\hline $\mathrm{C} 5-\mathrm{C} 6-\mathrm{C} 7-\mathrm{C} 8$ & $0.5(4)$ & $\mathrm{C} 6-\mathrm{C} 7-\mathrm{C} 16-\mathrm{C} 15$ & $-179.6(2)$ \\
\hline $\mathrm{C} 16-\mathrm{C} 7-\mathrm{C} 8-\mathrm{C} 9$ & $-0.9(3)$ & $\mathrm{C} 14-\mathrm{C} 15-\mathrm{C} 16-\mathrm{O} 1$ & $0.8(4)$ \\
\hline $\mathrm{C} 6-\mathrm{C} 7-\mathrm{C} 8-\mathrm{C} 9$ & $178.4(2)$ & $\mathrm{C} 10-\mathrm{C} 15-\mathrm{C} 16-\mathrm{O} 1$ & $-178.7(2)$ \\
\hline $\mathrm{C} 7-\mathrm{C} 8-\mathrm{C} 9-\mathrm{C} 10$ & $1.4(3)$ & $\mathrm{C} 14-\mathrm{C} 15-\mathrm{C} 16-\mathrm{C} 7$ & $-179.9(2)$ \\
\hline $\mathrm{C} 8-\mathrm{C} 9-\mathrm{C} 10-\mathrm{C} 11$ & $178.8(2)$ & $\mathrm{C} 10-\mathrm{C} 15-\mathrm{C} 16-\mathrm{C} 7$ & $0.6(3)$ \\
\hline $\mathrm{C} 8-\mathrm{C} 9-\mathrm{C} 10-\mathrm{C} 15$ & $-1.0(4)$ & & \\
\hline
\end{tabular}

Hydrogen-bond geometry $\left(A,{ }^{\circ}\right)$

$\mathrm{Cg} 3$ and $\mathrm{Cg} 4$ are the centroids of rings $\mathrm{C} 7-\mathrm{C} 10 / \mathrm{C} 15 / \mathrm{C} 16$ and $\mathrm{C} 10-\mathrm{C} 15$, respectively.

\begin{tabular}{lllll}
\hline$D-\mathrm{H}^{\cdots} A$ & $D-\mathrm{H}$ & $\mathrm{H} \cdots A$ & $D \cdots A$ & $D-\mathrm{H} \cdots A$ \\
\hline $\mathrm{C} 5-\mathrm{H} 5 B \cdots C g 4^{\mathrm{i}}$ & 0.99 & 2.69 & $3.664(3)$ & 167
\end{tabular}


Symmetry code: (i) $-x,-y+2, z-1 / 2$. 